\title{
Validación Diferencial y Discriminante del Cuestionario de Depresión para Hombres (CDH)
}

\section{Differential Discriminant Validation and Depression Questionnaire for Men (CDH)}

\author{
Constanza Londoño \\ Wenceslao Peñate \\ Manuel González \\ Universidad Católica de Colombia \\ Universidad de La Laguna, España
}

Rec (20 diciembre 2014) Acept (24 junio 2016)

\section{Resumen}

El objetivo de este estudio era evaluar la validez (diferencial/discriminante) y la sensibilidad y especificidad del Cuestionario de Depresión en Hombres. En la muestra clínica había 59 hombres y 59 mujeres entre 18 y 65 años diagnosticados previamente con depresión, que fueron comparados entre sí y con una muestra de controles con características sociodemográficas similares. Como medida de contraste estaba el concepto profesional y la Entrevista MINI de Depresión. Para los análisis psicométricos se usó el Modelo de Rasch desde la Teoría de Respuesta al Ítem y se hicieron análisis de covariación entre las medidas. Se concluye que el instrumento cuenta con un nivel alto de validez y confiabilidad y que tiene un nivel de sensibilidad y especificidad similar al de la escala de contraste.

Palabras clave: Depresión, validez, confiabilidad, sensibilidad y especificidad.

\begin{abstract}
The aim of this study was to assess the validity (differential/discriminant) and the sensitivity and specificity of Depression in Men Questionnaire. In the clinical sample, there were 59 men and 59 women between 18 and 65 years previously diagnosed with depression, which were compared with each other and with a sample of controls with similar demographic characteristics. As a contrast it was the professional concept and MINI interview Depression. For psychometric analyzes the Rasch model was used from the Item Response Theory and analysis of covariance between the measurements were made. It is concluded that the instrument has a high level of validity and reliability, and has a level of sensitivity and similar to the contrast scale specificity. Keywords: Depression, validity, reliability, sensitivity and specificity.
\end{abstract}




\section{Introducción}

La depresión es un problema mundial de salud pública que afecta el perfil general de salud, disminuye la productividad, se asocia a más de la mitad de pérdida de vidas por suicidio en varones jóvenes (Kilmartin, 2005) y al incremento de ira, hostilidad y conducta violenta relacionada con la depresión en los hombres (Besharat, Addolmananfi, Farahaniy Khodaii, 2011; Joormann y Gotlib, 2010; Rude, Chrisman, Denmark y Maestas, 2012; Worley et al., 2012).

A pesar de la evidencia contraria aún se afirma que no existen diferencias de género en los síntomas de depresión; sin tener en cuenta que un grupo de autores provenientes de distintas partes del mundo considera importante investigar acerca de las diferencias de síntomas entre hombres y mujeres (Brownhill, Wilhelm, Barclay y Schmied, 2005; Cochran y Rabinowitz, 2003; Mitendorfer-Rutz, 2006a y 2006b; Paykel, 1991; Pollack, Garbarino, Kindlon y Thompson, 1999; Rodié y Usall, 2001; Wilhelm, Boyce y Brownhill, 2004), y en consecuencia confieren alta relevancia al desarrollo de nuevos instrumentos sensibles a dichas diferencias.

Ya Álvarez y Londoño (2012) habían notado que los estudios DSM (Manual Diagnóstico y Estadístico de los Trastornos Mentales) se han realizado con muestras predominantemente femeninas y que, por tanto, los instrumentos de evaluación y diagnóstico derivados tienen sesgo real relacionado con el género (Galicia, Sánchez y Robles, 2009; Vargas, Ruiz, Hernández y Londoño, 2013). Ya Chang et al. (2008) habían encontrado serias diferencias en estadísticas epidemiológicas de la misma población, dependientes de las escalas usadas, ya que estos instrumentos no son sensibles al género; de este modo, puede sub diagnosticarse la depresión en población masculina; y para solucionar estas limitaciones es preciso que se desarrollen escalas específicas.

Para evaluar la depresión se usan medidas como el Beck Depression Inventory (BDI), la escala Hamilton y otros instrumentos validados con muestras eminentemente femeninas, ejemplo de ello son el estudio Nacional de Salud Mental en Colombia, los que hace la OMS periódicamente, la Encuesta Mundial de Salud Mental y otras encuestas internacionales (OMS, 2009; OPS, 2012) en las que se usa el BDI cuya sensibilidad en casos masculinos ha sido puesta en duda porque se considera que es reducida.

Para resolver esta limitación diagnóstica Álvarez y Londoño (2012) diseñaron y validaron en población general el Cuestionario de Depresión para Hombres (CDH) en el que incluyeron los síntomas reportados en estudios previos (Kilmartin, 2005; Möller, Heller y Paulus, 2006; Möller-Leimkühler y Yücel, 2010; Rodié y Usall, 2001). Para continuar con el proceso de diseño del instrumento y lograr los alcances diagnósticos esperados; el presente estudio tenía como objetivo evaluar la validez (diferencial/ discriminante) y otras condiciones psicométricas relacionadas con la sensibilidad y especificidad del CDH.

\section{Método}

En el presente estudio se realizaron análisis comparativos entre dos grupos clínicos y dos grupos no clínicos: G1: Grupo de hombres no clínicos; G2: Grupo de mujeres no clínicas; G3: Grupo de mujeres diagnosticadas con depresión; y G4: Grupo de hombres diagnosticados con depresión.

\section{Muestra}

En la muestra clínica, había 59 hombres y 59 mujeres entre 18 y 65 años diagnosticados previamente con depresión por un profesional psicólogo o psiquiatra bajo los criterios del DSM-IV, su juicio clínico y en algunos casos el uso de instrumentos reconfirmatorios (BDI-II, Escala Zung y Escala Hamilton); los profesionales trabajaban en distintos centros de atención ubicados en las 5 ciudades; en la muestra no clínica (G1 y G2) habían 54 hombres y 54 mujeres sin diagnóstico de depresión con características sociodemográficas comparables a las de los participantes ubicados en los grupos G3 y G4 (usuarias de servicios de consulta, sexo y género declarado, edad, región procedencia y nivel educativo), pero no mostraban indicadores de otro trastorno mental en la prueba de salud mental general Self Reporting Questionaire SQR 20 (Beusenberg y Orley, 1994) y que no presentaban depresión en la Entrevista MINI de Depresión (Sheehan et al., 1988).

Criterios de inclusión G4: 1) Hombres con diagnóstico confirmado de trastornos del espectro depresivo diagnosticados entre los meses de mayo a noviembre de 2013,2) sin tratamiento farmacológico (en Colombia el sistema de salud y las condiciones culturales limitan el acceso a la medicación aun en casos en los que es altamente recomendable).

Criterios de exclusión G4: 1) Diagnóstico de trastorno bipolar, 2) encontrarse en un periodo de remisión o recuperación, 3) no saber leer y escribir, 4) rehusar la participación y/o 5) mostrar indicadores de otros trastornos mentales del espectro psicótico. Los criterios básicos de inclusión para el grupo G2 eran los mismos del G3 solo que en este caso 
debían ser mujeres sin depresión; y para el G1 eran los mismos que para el grupo G4 solo que debían ser hombres sin depresión.

\section{Instrumentos}

Cuestionario de Depresión en Hombres (CDH) de Álvarez y Londoño (2012): compuesto por 40 ítems con opciones de respuesta en escala Likert, que miden síntomas considerados característicos de depresión en hombres. La puntuación total de los ítems se da en cortes distribuidos así: 1-28 sin depresión, 29-34 depresión leve, 35-40 depresión moderada, y mayor de 41 depresión severa. El índice de evaluación de confiabilidad entre .87 y .90 , un nivel de separación de 2.61 que indica la existencia de un grado alto de confiabilidad (Vargas et al., 2013). La escala evalúa 6 dimensiones, que son autoimagen negativa con un índice de fiabilidad de .85 , ideación suicida con .93 , pobre imagen social con .87 , afecto negativo de .94 , desesperanza de .80 y evitación de .75 .
Escalas relacionadas con trastornos del estado de ánimo (episodio depresivo mayor, trastorno distímico y riesgo de suicidio) del Mini International Neuropsychiatric Interview (MINI) utilizada como prueba de oro de referencia en la validación del CDH y como estrategia de selección de los casos incluidos en los grupos no clínicos. El MINI es una entrevista diagnóstica estructurada de breve duración que explora de manera independiente los principales trastornos psicopatológicos del eje I del DMSIV y el CIE-10. La entrevista goza de un buen nivel de discriminación clínica cuando es realizada por personal entrenado, soportado en diversos estudios y en la confiabilidad test-retest que está entre .76 y .93 , y puede ser aplicada por factores ya que existe independencia entre ellos (Sheehan et al., 1988).

Cuestionario de Autorreporte de Sintomas SQR (1980): creado por la OMS y adaptado para población colombiana (Beusenberg y Orley, 1994), es usado para tamizar problemas de salud mental, la muestra base incluyó países latinoamericanos entre los que Colombia estaba incluida. El cuestionario consta de 30 preguntas que evalúan trastornos

Tabla 1. Descripción de las características sociodemográficas en muestra total y por grupos

\begin{tabular}{|c|c|c|c|c|c|c|c|c|c|c|}
\hline \multirow{3}{*}{ Variables } & \multirow{3}{*}{$\begin{array}{l}\text { Total } \\
\mathrm{f}\end{array}$} & \multirow{2}{*}{\multicolumn{2}{|c|}{$\begin{array}{l}\text { Hombres } \\
\text { No clínicos }\end{array}$}} & & \multicolumn{2}{|l|}{ Mujeres } & \multirow{2}{*}{ Mujeres } & & \multicolumn{2}{|c|}{ Hombres } \\
\hline & & & & \multicolumn{2}{|c|}{ No clínicas } & Clínicas & & \multicolumn{3}{|c|}{ Clínicos } \\
\hline & & $\%$ & $\mathrm{f}$ & $\%$ & $\mathrm{f}$ & $\%$ & $\mathrm{f}$ & $\%$ & $\mathrm{f}$ & $\%$ \\
\hline \multicolumn{11}{|c|}{ Nivel educativo } \\
\hline Primaria & 6 & 2.7 & 1 & 1.8 & 2 & 3.7 & 1 & 1.6 & 2 & 3.3 \\
\hline Bachillerato & 24 & 11 & 4 & 7.4 & 5 & 9.2 & 7 & 11.8 & 6 & 10.1 \\
\hline Técnico & 18 & 8.2 & 3 & 5.5 & 5 & 9.2 & 4 & 6.7 & 7 & 11.8 \\
\hline Universitario & 146 & 66.9 & 37 & 68.5 & 35 & 64.8 & 36 & 61 & 37 & 62.7 \\
\hline Postgrados & 32 & 14.6 & 6 & 11.1 & 12 & 22.2 & 6 & 10.1 & 6 & 10.1 \\
\hline \multicolumn{11}{|l|}{ Estrato } \\
\hline 1 & 10 & 4.5 & 1 & 1.8 & 1 & 1.8 & 2 & 3.3 & 5 & 8.4 \\
\hline 2 & 69 & 31.6 & 12 & 22.2 & 20 & 37 & 21 & 35.5 & 17 & 28.8 \\
\hline 3 & 94 & 43.1 & 27 & 50 & 24 & 44.4 & 21 & 35.5 & 22 & 37.2 \\
\hline 4 & 45 & 20.6 & 12 & 22.2 & 13 & 24 & 12 & 20.3 & 12 & 20.3 \\
\hline 5 & 6 & 2.7 & 2 & 3.7 & 1 & 1.8 & 1 & 1.6 & 1 & 1.6 \\
\hline 6 & 2 & 0.9 & 0 & 0 & 0 & 0 & 2 & 3.3 & 2 & 3.3 \\
\hline \multicolumn{11}{|l|}{ Estado civil } \\
\hline Soltero & 157 & 72 & 36 & 66.6 & 41 & 75.9 & 41 & 69.4 & 39 & 66.1 \\
\hline Casado & 47 & 21.5 & 10 & 18.5 & 13 & 24 & 11 & 18.6 & 13 & 22 \\
\hline Separado & 15 & 6.8 & 6 & 11.1 & 4 & 7.4 & 1 & 1.6 & 4 & 6.7 \\
\hline Viudo & 7 & 3.2 & 2 & 3.7 & 1 & 1.8 & 1 & 1.6 & 3 & 5 \\
\hline
\end{tabular}

Nota: f: Frecuencia; \%: Porcentaje 
depresión, angustia entre otros de leve y moderada intensidad (preguntas 1 y 21), trastornos psicóticos, trastorno compulsivo, alcoholismo o abuso de alcohol. Se considera de alta sensibilidad y posee una confiabilidad de .89. (Gonçalves, Stein y Kapczinski, 2008).

\section{Procedimiento}

En primer lugar 8 profesionales psicólogos identificaron los casos remitidos por los profesionales psicólogos y psiquiatras y verificaron el cumplimiento de los criterios de inclusión del estudio; una vez identificado el caso, se realizaba el procedimiento para lograr el consentimiento informado. La entrevista fue realizada por un asistente de investigación estudiante de postgrado, quien aplicó el apartado de Trastornos del Estado de Ánimo del MINI. Si detectaba señales de otras problemáticas importantes, realizaba la entrevista completa. Para evitar sesgos los entrevistadores no discutieron el diagnóstico con el profesional responsable del caso.

\section{Resultados}

La mayor parte de la muestra tenía estudios universitarios (64.2\%), era de nivel socioeconómico medio bajo y bajo (estrato 3 y 2) $(72.16 \%)$, en su mayoría solteros (72 \%) (Tabla 1).

Para analizar la confiabilidad, validez, estructura (dimensionalidad), discriminación y determinación de los puntos de corte se usó el Modelo de Rasch desde la Teoría de Respuesta al Ítem y para evaluar el funcionamiento diferencial de los ítems se utilizó el índice Mantel Haenszel (MH). La validez concurrente se analizó a partir de la observación de la covariación (linealidad, inclinación de la pendiente) y del cálculo de los índices de correlación de Pearson, con un índice de significación de p menor o igual a .05 .

Se identificaron 4 casos con comportamiento atípico que dieron una respuesta constante en el $\mathrm{CDH}$, y se encontró que el instrumento tiene un nivel de fiabilidad alto reflejado en los análisis realizados al excluir los casos atípicos. El índice de separación (4.19) reflejo un nivel de ajuste apropiado (Tabla 2).

Con el objetivo de evitar fallas de funcionamiento del cuestionario al ser respondido por las mujeres con depresión, se analizan el funcionamiento de los ítems en el G4 y se evidencia en general que estos cumplen con la condición de tener puntajes entre 1 y 1.3 en el INFIT, y puntajes menores a 2.0 en el OUTFIT, con un mejor ajuste que el logrado con ambos grupos clínicos (Tabla 3).

Para reconfirmar que no existan fallas de funcionamiento del Cuestionario al ser respondido por las mujeres clínicas, se analizan el funcionamiento de los ítems los participantes de G3 y G4, y se evidencia los ítems cumplen con la condición de tener puntajes entre 1 y 1.3 en el INFIT, y puntajes menores a 2.0 en el OUTFIT (Tabla 4). Posteriormente se analizó el funcionamiento de los ítems únicamente en el G4 y se evidenció que estos cumplían con la condición de tener puntajes entre 1 y 1.3 en el INFIT, y puntajes menores a 2.0 en el OUTFIT, a excepción de los ítems 7, 22, 25 y 28, en los que se evidenciaron problemas de funcionamiento. Se entrevistaron 15 participantes acerca de los ítems con funcionamiento anómalo y se concluyó que había fallas de redacción.

Tabla 2. Resumen de 223 medidas no extremas y cálculos de índices fiabilidad como separación, ajuste y consistencia interna del CDH.

\begin{tabular}{lccccccc}
\hline & Puntaje total & İtems contados & Medida & Error del & \multicolumn{2}{c}{ INFIT } & OUTFIT \\
\cline { 6 - 8 } & & & & Modelo & MNSQ & MNSQ \\
\hline Media & 73.5 & 40.0 & -0.53 & 0.26 & 1.00 & 1.01 \\
DT & 22.2 & 0 & 1.30 & 0.06 & 0.52 & 0.59 \\
Máx. & 138.0 & 40.0 & 2.56 & 0.49 & 3.82 & 4.21 \\
Mín. & 40.0 & 40.0 & -4.03 & 0.19 & 0.10 & 0.07 \\
& & & & & & & \\
Real RSME & 0.30 & de real & 1.26 & Separación & 4.19 & Confiabilidad & 0.95 \\
Model RSME & 0.27 & de real & 1.27 & Separación & 4.62 & Confiabilidad & 0.96 \\
de participantes & 0.08 & & & & & & \\
$\mathrm{~N}$ & 223 & & & & & & \\
\hline
\end{tabular}

Nota: Excluidos los puntajes mínimos extremos 3 participantes 1.3\%; DT: Desviación típica; MNSQ: Mínimos Cuadrados Normados;RSME: Raíz Cuadrada del Error Cuadrático Medio;INFIT: Ajuste próximo; OUFIT: Ajuste lejano. 
Tabla 3. Funcionamiento individual de los items del Cuestionario de Depresión en Hombres con INFIT (ajuste próximo) y OUTFIT (Ajuste lejano) en 59 casos

\begin{tabular}{|c|c|c|c|c|c|c|c|c|c|c|c|}
\hline \multirow[t]{2}{*}{ Ítem } & \multirow[t]{2}{*}{ PT } & \multirow[t]{2}{*}{$X$} & \multirow[t]{2}{*}{$\mathrm{XM}$} & \multirow{2}{*}{$\begin{array}{c}\text { INFIT } \\
\text { MNSQ }\end{array}$} & \multirow{2}{*}{$\begin{array}{c}\text { OUTFIT } \\
\text { MNSQ }\end{array}$} & \multicolumn{2}{|c|}{ PTM MEDIDA } & \multirow[t]{2}{*}{ OBS EXAC \% } & \multirow[t]{2}{*}{ МATCH EXP \% } & \multirow[t]{2}{*}{ ESTIM DISCR } & \multirow[t]{2}{*}{ p-valor } \\
\hline & & & & & & CORR & EXP & & & & \\
\hline 1 & 148 & 0.20 & 0.24 & 0.95 & 1.02 & 0.55 & 0.54 & 61.1 & 58.2 & 1.04 & 2.74 \\
\hline 2 & 105 & -0.34 & 0.20 & 0.91 & 0.91 & 0.65 & 0.60 & 61.1 & 51.9 & 1.10 & 1.94 \\
\hline 3 & 121 & -0.11 & 0.17 & 1.28 & 1.40 & 0.51 & 0.66 & 38.9 & 45.1 & 0.45 & 2.24 \\
\hline 4 & 116 & -0.40 & 0.17 & 0.84 & 0.77 & 0.74 & 0.67 & 53.7 & 44.9 & 1.25 & 2.15 \\
\hline 5 & 138 & -0.90 & 0.20 & 1.40 & 1.38 & 0.48 & 0.62 & 50.0 & 56.0 & 0.70 & 2.56 \\
\hline 6 & 152 & -1.31 & 0.22 & 1.37 & 1.35 & 0.34 & 0.56 & 48.1 & 56.3 & 0.56 & 2.81 \\
\hline 7 & 145 & -1.66 & 0.17 & 1.18 & 1.20 & 0.56 & 0.65 & 51.9 & 45.6 & 0.73 & 2.69 \\
\hline 8 & 124 & -0.23 & 0.19 & 0.93 & 0.90 & 0.65 & 0.62 & 59.3 & 52.5 & 1.08 & 2.30 \\
\hline 9 & 126 & -0.33 & 0.20 & 1.00 & 0.96 & 0.61 & 0.61 & 68.5 & 54.9 & 1.00 & 2.33 \\
\hline 10 & 100 & 0.61 & 0.19 & 1.01 & 0.95 & 0.63 & 0.63 & 57.4 & 53.6 & 1.02 & 1.85 \\
\hline 11 & 83 & -0.49 & 0.19 & 1.78 & 1.86 & 0.30 & 0.60 & 53.7 & 63.7 & 0.51 & 1.54 \\
\hline 12 & 107 & -0.89 & 0.18 & 0.88 & 0.75 & 0.71 & 0.66 & 63.0 & 48.7 & 1.11 & 1.98 \\
\hline 13 & 120 & -0.01 & 0.18 & 1.15 & 1.27 & 0.57 & 0.63 & 48.1 & 48.9 & 0.79 & 2.22 \\
\hline 14 & 112 & 0.26 & 0.20 & 1.05 & 1.02 & 0.58 & 0.59 & 51.9 & 54.7 & 0.97 & 2.07 \\
\hline 15 & 149 & -1.46 & 0.22 & 0.85 & 0.85 & 0.65 & 0.56 & 61.1 & 56.5 & 1.19 & 2.76 \\
\hline 16 & 99 & 0.64 & 0.19 & 0.57 & 0.54 & 0.80 & 0.63 & 64.8 & 53.8 & 1.47 & 1.83 \\
\hline 17 & 115 & 0.00 & 0.18 & 1.66 & 1.92 & 0.37 & 0.66 & 48.1 & 45.6 & 0.01 & 2.13 \\
\hline 18 & 127 & -0.28 & 0.20 & 0.82 & 0.83 & 0.69 & 0.60 & 63.0 & 54.9 & 1.21 & 2.35 \\
\hline 19 & 82 & 1.38 & 0.22 & 0.70 & 0.74 & 0.67 & 0.55 & 70.4 & 65.7 & 1.22 & 1.52 \\
\hline 20 & 110 & 0.45 & 0.21 & 0.76 & 0.74 & 0.72 & 0.58 & 61.1 & 54.4 & 1.33 & 2.04 \\
\hline 21 & 125 & -0.05 & 0.20 & 0.87 & 0.88 & 0.67 & 0.59 & 66.7 & 55.6 & 1.19 & 2.31 \\
\hline 22 & 127 & -0.15 & 0.19 & 1.73 & 1.75 & 0.23 & 0.60 & 38.9 & 52.9 & 0.01 & 2.35 \\
\hline 23 & 106 & 0.34 & 0.18 & 0.76 & 0.70 & 0.75 & 0.66 & 55.6 & 51.8 & 1.24 & 1.96 \\
\hline 24 & 131 & -1.37 & 0.18 & 1.15 & 1.17 & 0.57 & 0.64 & 53.7 & 49.6 & 0.81 & 2.43 \\
\hline 25 & 92 & 0.70 & 0.19 & 0.60 & 0.66 & 0.75 & 0.61 & 70.4 & 59.2 & 1.35 & 1.70 \\
\hline 26 & 135 & -0.69 & 0.21 & 0.62 & 0.62 & 0.80 & 0.59 & 72.2 & 57.1 & 1.43 & 2.50 \\
\hline 27 & 125 & -0.12 & 0.21 & 1.43 & 1.42 & 0.33 & 0.57 & 53.7 & 58.8 & 0.53 & 2.31 \\
\hline 28 & 107 & -0.43 & 0.19 & 1.60 & 1.53 & 0.34 & 0.61 & 44.4 & 50.9 & 0.21 & 1.98 \\
\hline 29 & 117 & 0.04 & 0.18 & 0.72 & 0.70 & 0.77 & 0.64 & 55.6 & 48.6 & 1.4 & 2.17 \\
\hline 30 & 118 & -0.40 & 0.18 & 0.91 & 0.85 & 0.69 & 0.64 & 55.6 & 49.0 & 1.17 & 2.19 \\
\hline 31 & 129 & -0.38 & 0.19 & 0.85 & 0.82 & 0.70 & 0.61 & 61.1 & 53.5 & 1.22 & 2.39 \\
\hline 32 & 95 & 0.74 & 0.19 & 0.63 & 0.52 & 0.79 & 0.63 & 74.1 & 57.9 & 1.40 & 1.76 \\
\hline 33 & 101 & 0.70 & 0.20 & 0.89 & 0.84 & 0.66 & 0.60 & 46.3 & 52.2 & 1.16 & 1.87 \\
\hline 34 & 111 & 0.34 & 0.19 & 0.82 & 0.79 & 0.71 & 0.62 & 59.3 & 49.5 & 1.26 & 2.06 \\
\hline 35 & 88 & 0.38 & 0.23 & 0.84 & 0.76 & 0.65 & 0.57 & 61.1 & 60.8 & 1.22 & 1.63 \\
\hline 36 & 85 & 1.09 & 0.21 & 0.72 & 0.74 & 0.69 & 0.59 & 66.7 & 63.9 & 1.22 & 1.57 \\
\hline 37 & 101 & 0.52 & 0.18 & 0.92 & 1.00 & 0.68 & 0.66 & 51.9 & 55.1 & 1.04 & 1.87 \\
\hline 38 & 92 & 0.28 & 0.23 & 1.00 & 1.07 & 0.54 & 0.55 & 68.5 & 59.1 & 0.97 & 1.70 \\
\hline 39 & 86 & 1.07 & 0.20 & 0.78 & 0.75 & 0.67 & 0.60 & 57.4 & 63.1 & 1.15 & 1.59 \\
\hline 40 & 97 & 0.68 & 0.19 & 0.72 & 0.78 & 0.72 & 0.62 & 70.4 & 55.2 & 1.29 & 1.80 \\
\hline$X$ & 113.7 & 0.00 & 0.20 & 0.99 & 0.99 & & 58.0 & 54.2 & & & \\
\hline DT & 18.8 & 0.70 & 0.01 & 0.31 & 0.35 & & 8.7 & 5.2 & & & \\
\hline
\end{tabular}

Nota: PT: Puntaje Total;TC: Total Casos;X: Media; DT: Desviación típica;XM: Media del Modelo;PTMA: Correlación Producto Momento;OBS EXAC: Observaciones Exactas;MACTH EXAC: MACTH exactas;ESTIM DISCRIM: Estimación de la discriminación;P VALOR: Estimación de la probabilidad; MNSQ: Mínimos Cuadrados Normados;RSME: Raíz Cuadrada del Error Cuadrático Medio; INFIT: Ajuste próximo;OUTFIT: Ajuste lejano 
Tabla 4. Funcionamiento individual de los ítems del Cuestionario de Depresión en Hombres.

\begin{tabular}{|c|c|c|c|c|c|c|c|c|c|c|c|c|}
\hline \multirow[t]{2}{*}{ Ítem } & \multirow[t]{2}{*}{ PT } & \multirow[t]{2}{*}{$\mathrm{TC}$} & \multirow[t]{2}{*}{$\mathrm{X}$} & \multirow[t]{2}{*}{ XM } & \multirow{2}{*}{$\begin{array}{c}\text { INFIT } \\
\text { MNSQ }\end{array}$} & \multirow{2}{*}{$\begin{array}{l}\text { OUTFIT } \\
\text { MNSQ }\end{array}$} & \multicolumn{2}{|c|}{ PTM MEDIDA } & \multirow{2}{*}{$\begin{array}{c}\text { OBS. } \\
\text { EXAC } \%\end{array}$} & \multirow{2}{*}{$\begin{array}{c}\text { МATCH } \\
\text { EXP \% }\end{array}$} & \multirow{2}{*}{$\begin{array}{l}\text { ESTIM. } \\
\text { DISCR }\end{array}$} & \multirow[t]{2}{*}{$\mathrm{P}$} \\
\hline & & & & & & & CORR & EXP & & & & \\
\hline 1 & 557 & 226 & -0.62 & 0.11 & 0.87 & 0.86 & 0.67 & 0.60 & 64.6 & 60 & 1.15 & 2.46 \\
\hline 2 & 388 & 226 & -0.42 & 0.10 & 0.91 & 0.86 & 0.64 & 0.62 & 65.0 & 58.1 & 1.07 & 1.72 \\
\hline 3 & 417 & 226 & -1.17 & 0.09 & 1.16 & 1.37 & 0.58 & 0.63 & 54.7 & 54.1 & 0.78 & 1.85 \\
\hline 4 & 416 & 226 & -0.86 & 0.09 & 0.81 & 0.77 & 0.68 & 0.63 & 60.1 & 53.8 & 1.17 & 1.84 \\
\hline 5 & 499 & 226 & -0.01 & 0.10 & 1.06 & 1.05 & 0.61 & 0.63 & 59.6 & 58.3 & 0.96 & 2.21 \\
\hline 6 & 529 & 226 & -0.14 & 0.10 & 0.86 & 0.85 & 0.71 & 0.64 & 57.8 & 54.4 & 1.18 & 2.34 \\
\hline 7 & 525 & 226 & -1.76 & 0.09 & 1.29 & 1.33 & 0.60 & 0.67 & 51.1 & 49.2 & 0.65 & 2.32 \\
\hline 8 & 463 & 226 & -1.07 & 0.10 & 0.93 & 0.85 & 0.67 & 0.64 & 66.4 & 60.5 & 1.06 & 2.05 \\
\hline 9 & 454 & 226 & 0.41 & 0.10 & 0.90 & 0.85 & 0.70 & 0.65 & 70.0 & 55.7 & 1.17 & 2.01 \\
\hline 10 & 382 & 226 & 1.00 & 0.10 & 0.83 & 0.92 & 0.68 & 0.63 & 64.6 & 57.9 & 1.16 & 1.69 \\
\hline 11 & 307 & 226 & -0.00 & 0.12 & 1.58 & 1.62 & 0.39 & 0.56 & 63.7 & 71.4 & 0.63 & 1.36 \\
\hline 12 & 415 & 226 & -0.95 & 0.09 & 0.87 & 0.74 & 0.67 & 0.63 & 57.8 & 53.6 & 1.13 & 1.84 \\
\hline 13 & 411 & 226 & -1.09 & 0.09 & 1.03 & 1.15 & 0.61 & 0.63 & 53.4 & 54.6 & 0.92 & 1.82 \\
\hline 14 & 390 & 226 & -0.79 & 0.10 & 1.08 & 1.05 & 0.59 & 0.61 & 56.5 & 57.9 & 0.91 & 1.73 \\
\hline 15 & 542 & 226 & -1.71 & 0.10 & 1.01 & 1.00 & 0.67 & 0.67 & 57.0 & 56.8 & 1.00 & 2.40 \\
\hline 16 & 351 & 226 & 1.27 & 0.10 & 0.70 & 0.72 & 0.69 & 0.60 & 70.9 & 63.7 & 1.24 & 1.55 \\
\hline 17 & 399 & 226 & 0.86 & 0.10 & 1.34 & 1.35 & 0.52 & 0.64 & 52.5 & 56.2 & 0.62 & 1.77 \\
\hline 18 & 440 & 226 & -1.17 & 0.09 & 0.92 & 0.91 & 0.66 & 0.64 & 59.2 & 54.1 & 1.08 & 1.95 \\
\hline 19 & 312 & 226 & 1.74 & 0.12 & 0.65 & 0.59 & 0.66 & 0.56 & 74.4 & 72.4 & 1.22 & 1.38 \\
\hline 20 & 401 & 226 & 1.08 & 0.10 & 0.85 & 0.79 & 0.69 & 0.62 & 65.5 & 58.0 & 1.21 & 1.77 \\
\hline 21 & 477 & 226 & 0.34 & 0.10 & 0.82 & 0.81 & 0.72 & 0.63 & 65.5 & 56.4 & 1.23 & 2.11 \\
\hline 22 & 447 & 226 & -1.19 & 0.10 & 1.99 & 2.20 & 0.35 & 0.64 & 38.1 & 54.2 & -.14 & 1.98 \\
\hline 23 & 380 & 226 & 0.96 & 0.10 & 0.77 & 0.91 & 0.70 & 0.63 & 68.2 & 58.1 & 1.20 & 1.68 \\
\hline 24 & 426 & 226 & -1.15 & 0.09 & 0.97 & 0.88 & 0.65 & 0.64 & 62.3 & 53.5 & 1.07 & 1.88 \\
\hline 25 & 345 & 226 & -0.60 & 0.10 & 1.08 & 1.62 & 0.55 & 0.59 & 68.2 & 64.7 & 0.89 & 1.53 \\
\hline 26 & 488 & 226 & 0.08 & 0.10 & 069 & 0.69 & 0.78 & 0.65 & 68.2 & 55.8 & 1.37 & 2.16 \\
\hline 27 & 444 & 226 & -1.14 & 0.10 & 1.34 & 1.29 & 0.55 & 0.64 & 54.3 & 56.7 & 0.67 & 1.96 \\
\hline 28 & 367 & 226 & -0.82 & 0.10 & 1.78 & 2.24 & 0.40 & 0.60 & 53.4 & 59.3 & 0.34 & 1.62 \\
\hline 29 & 413 & 226 & -1.06 & 0.10 & 0.71 & 0.69 & 0.70 & 0.63 & 61.9 & 55.3 & 1.29 & 1.83 \\
\hline 30 & 380 & 226 & 1.05 & 0.10 & 0.94 & 0.82 & 0.67 & 0.63 & 66.8 & 57.9 & 1.13 & 1.68 \\
\hline 31 & 449 & 226 & -1.18 & 0.10 & 1.01 & 1.02 & 0.64 & 0.64 & 57.8 & 55.0 & 0.98 & 1.99 \\
\hline 32 & 343 & 226 & 1.28 & 0.10 & 0.78 & 0.77 & 0.67 & 0.61 & 71.7 & 66.1 & 1.15 & 1.52 \\
\hline 33 & 386 & 226 & 0.98 & 0.10 & 0.87 & 0.90 & 0.68 & 0.63 & 58.3 & 57.2 & 1.13 & 1.71 \\
\hline 34 & 387 & 226 & 1.12 & 0.10 & 0.81 & 0.74 & 0.70 & 0.62 & 64.6 & 58.1 & 1.24 & 1.71 \\
\hline 35 & 330 & 226 & 1.79 & 0.12 & 1.03 & 0.99 & 0.56 & 0.57 & 65.5 & 67.8 & 0.98 & 1.46 \\
\hline 36 & 314 & 226 & 1.45 & 0.11 & 0.54 & 0.39 & 0.69 & 0.57 & 81.2 & 73.2 & 1.26 & 1.39 \\
\hline 37 & 354 & 226 & -0.67 & 0.10 & 0.93 & 0.90 & 0.60 & 0.60 & 65.5 & 63.4 & 1.00 & 1.57 \\
\hline 38 & 367 & 226 & 1.37 & 0.11 & 1.26 & 1.26 & 0.51 & 0.61 & 58.3 & 60.2 & 0.73 & 1.62 \\
\hline 39 & 325 & 226 & 1.44 & 0.11 & 0.64 & 0.65 & 0.67 & 0.58 & 72.6 & 70.6 & 1.20 & 1.44 \\
\hline 40 & 368 & 226 & 1.36 & 0.10 & 0.83 & 0.90 & 0.67 & 0.62 & 65.0 & 60.2 & 1.14 & 1.63 \\
\hline$X$ & 409.7 & 226. & 0.00 & 0.10 & 0.99 & -0.1 & & 62.3 & 59.1 & & & \\
\hline DT & 64.0 & 0 & 1.08 & 0.00 & 0.29 & 2.7 & & 7.6 & 5.6 & & & \\
\hline
\end{tabular}

Nota: Pt: Puntaje Total; Tc: Total Casos; X: Dificultad; DT: Desviación Estándar; XML: Error de Medición;

Ptma: Correlación producto momento; Obs. Exac: Observaciones Exactas; MACTH Exac: MACTH exacto; Estim. Discrim: Estimación discriminante; p: Estimación de la probabilidad; MNSQ: Mínimos Cuadrados Normados; INFIT: Ajuste Próximo;

OUTFIT: Ajuste Lejano 
Tabla 5. Componentes o factores contenidos en el Cuestionario de Depresión en Hombres

\begin{tabular}{cccccc}
\hline Dimensiones & Denominación & Ítems & Media & DT & Confiabilidad \\
\hline 1 & Autoimagen negativa & $40,23,10,32$ & 6.52 & 2.92 & 0.85 \\
2 & Ideación Suicida & $36,37,19,39$ & 5.77 & 2.98 & 0.93 \\
3 & Pobre Imagen Social & $33,29,34,30,38,31$ & 10.54 & 4.05 & 0.87 \\
4 & Afecto negativo & $1,4,5,6,8,9,12,13,14,15,18,21$, & 30 & 9.43 & 0.94 \\
& & $24,25,26$ & & & \\
5 & Desesperanza & $2,3,16$ & 5.09 & 2.21 & 0.80 \\
6 & Evitación & $7,11,17,20,22,27,28,35$ & 14.25 & 4.21 & 0.75 \\
\hline
\end{tabular}

Nota: DT: Desviación típica.

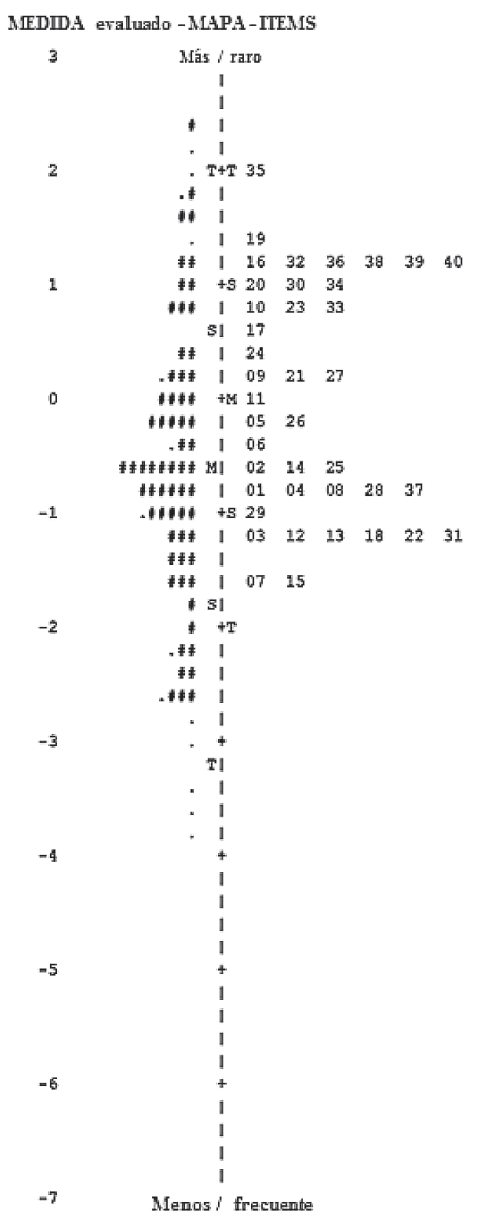

Nota: Cada participante: \#; Cada ítem: 1,40. Entrada participante: 226. Entrada ítems: 40

Figura 1. Diagrama de distribución de items y participantes del $\mathrm{CDH}$.
En el primer contraste la varianza explicada por la medida fue de $56.6 \%$. Las preguntas introducían el 30.7\% mayor que la varianza no explicada de $3.2 \%$; sin embargo, la varianza no explicada sobrepasó el límite establecido de 2.0 que indicaba la existencia de dimensiones; por ello se realizó un análisis dimensional con un criterio límite de 2.0 y de Loading de 0.45 de acuerdo con los criterios teóricos que podrían explicar la agrupación de los síntomas incluidos en el constructo depresión. Las preguntas se diferenciaban en 6 dimensiones de segundo orden: autoimagen negativa; ideación suicida; pobre imagen social, afecto negativo, desesperanza; y evitación (Tabla 5).

La distribución en el diagrama de los ítems y los participantes se encontraban entre 3 y -4 logits de forma más o menos paralela, lo que indica que se logró una alta comprensión de los ítems y alta correspondencia entre los mismos y el grado en el que se presentaba el constructo, es decir, que estos son discriminativos respecto de los puntajes reportados en la prueba (Figura 1).

En el análisis de funcionamiento diferencial de ítems respecto del género se contrastan las posibles diferencias teniendo en cuenta que el valor de la diferencia (Dif contrast) debía ser mayor a .5, la probabilidad de Rasch Welch fuera inferior a $0.5 \mathrm{y}$ la probabilidad Mantel Haenzel fuera inferior a .05 (Tabla 6).

Se encontró que los ítems 12, 17, 28, 30 y 22 tenían una probabilidad distinta de ser respondidos por los grupos G3 y G4, que se expresa en que los ítems 12 y 17 son más sensibles a mostrar la depresión en mujeres y los ítems 22, 28 y 30 en hombres. Asimismo, al comparar el G1 y el G2 se encuentra que los ítems 11 y 17 tienen diferente probabilidad de ser contestados o son más sensibles para mostrar un nivel alto de depresión (Tabla 7). 
Tabla 6. Análisis del funcionamiento diferencial de los items respecto del género en hombres deprimidos $(n=59)$ y mujeres deprimidas $(n=59)$

\begin{tabular}{|c|c|c|c|c|c|c|c|c|}
\hline \multirow{2}{*}{$\begin{array}{c}\text { Participante } \\
\text { Grupo }\end{array}$} & \multirow{2}{*}{$\begin{array}{l}\text { Diferencia } \\
\text { de medias }\end{array}$} & \multirow{2}{*}{$\begin{array}{c}\text { Diferencia } \\
\text { measure1 }\end{array}$} & \multirow{2}{*}{$\begin{array}{l}\text { Diferencia del } \\
\text { contraste }\end{array}$} & \multicolumn{3}{|c|}{ Rasch Welch } & \multicolumn{2}{|c|}{ Mantel Haenzel } \\
\hline & & & & $\mathrm{T}$ & d.f & Prob. & Chi & Prob. \\
\hline 3 & -1.08 & -1.15 & 0.07 & 0.23 & 108 & 0.81 & 0.16 & 0.68 \\
\hline 3 & -0.60 & -0.30 & -0.30 & -1.10 & 108 & 0.27 & 1.60 & 0.20 \\
\hline 3 & -0.97 & -1.19 & 0.21 & 9.90 & 108 & 0.37 & 0.09 & 0.76 \\
\hline 3 & -0.09 & 0.29 & -0.39 & -1.58 & 108 & 0.11 & 1.96 & 0.16 \\
\hline 3 & -0.44 & -0.48 & 0.41 & 0.15 & 108 & 0.88 & 0.27 & 0.59 \\
\hline 3 & -0.85 & -0.81 & -0.03 & -0.12 & 108 & 0.90 & 1.86 & 0.17 \\
\hline 3 & -1.43 & -1.53 & 0.10 & 0.42 & 108 & 0.67 & 0.24 & 0.61 \\
\hline 3 & -0.75 & -0.94 & 0.19 & 0.70 & 108 & 0.48 & 0.36 & 0.54 \\
\hline 3 & -0.22 & 0.01 & -0.22 & -0.83 & 108 & 0.40 & 0.20 & 0.65 \\
\hline 3 & 0.29 & 0.74 & -0.44 & -1.74 & 108 & 0.08 & 3.33 & 0.06 \\
\hline 3 & 0.25 & -0.05 & 0.30 & 1.00 & 107 & 0.32 & 2.21 & 0.13 \\
\hline 3 & -1.19 & -0.56 & -0.62 & -2.59 & 108 & 0.01 & 3.29 & 0.06 \\
\hline 3 & 0.37 & 0.19 & 0.18 & 0.73 & 108 & 0.46 & 1.07 & 0.30 \\
\hline 3 & -0.45 & -0.92 & 0.46 & 1.67 & 108 & 0.09 & 2.72 & 0.09 \\
\hline 3 & -1.65 & -1.42 & -0.23 & -0.79 & 108 & 0.43 & 0.04 & 0.83 \\
\hline 3 & 9.93 & 0.84 & 0.08 & 0.32 & 108 & 0.74 & 0.06 & 0.98 \\
\hline 3 & 0.84 & 0.32 & 0.52 & 2.00 & 107 & 0.04 & 2.28 & 0.13 \\
\hline 3 & -1.03 & -1.20 & 0.17 & 0.66 & 108 & 0.50 & 0.53 & 0.46 \\
\hline 3 & 1.17 & 1.42 & -0.24 & -0.87 & 108 & 0.38 & 2.27 & 0.13 \\
\hline 3 & 0.54 & 0.86 & -0.32 & -1.14 & 108 & 0.25 & 0.26 & 0.60 \\
\hline 3 & -0.41 & 0.11 & -0.53 & -1.87 & 108 & 0.06 & 2.01 & 0.15 \\
\hline 3 & 1.08 & 0.20 & 0.88 & 3.27 & 107 & 0.01 & 4.96 & 0.02 \\
\hline 3 & 0.67 & 0.53 & 0.13 & 0.55 & 108 & 0.58 & 0.03 & 0.08 \\
\hline 3 & -1.14 & -1.14 & 0.02 & 0.01 & 108 & 0.99 & 0.08 & 0.77 \\
\hline 3 & -0.68 & -0.33 & -0.34 & -1.36 & 108 & 0.17 & 0.09 & 0.75 \\
\hline 3 & -0.62 & -0.46 & -0.16 & -0.58 & 108 & 0.56 & 0.52 & 0.47 \\
\hline 3 & 0.20 & 0.14 & 0.06 & 0.23 & 108 & 0.81 & 0.16 & 0.68 \\
\hline 3 & -1.14 & -0.81 & 0.62 & 2.23 & 107 & 0.02 & 5.55 & 0.01 \\
\hline 3 & 0.39 & 0.16 & 0.22 & 0.86 & 108 & 0.38 & 0.73 & 0.39 \\
\hline 3 & 1.05 & 0.27 & 0.77 & 2.88 & 107 & 0.04 & 3.03 & 0.08 \\
\hline 3 & -1.03 & -1.17 & 0.14 & 0.54 & 108 & 0.59 & 0.77 & 0.37 \\
\hline 3 & 1.01 & 0.90 & 0.11 & 0.42 & 108 & 0.67 & 1.14 & 0.28 \\
\hline 3 & 0.34 & 0.70 & -0.36 & -1.44 & 108 & 0.15 & 0.83 & 0.36 \\
\hline 3 & 0.76 & 0.65 & 0.10 & 0.40 & 108 & 0.69 & 0.57 & 0.44 \\
\hline 3 & 1.42 & 1.35 & 0.06 & 0.24 & 108 & 0.81 & 0.10 & 0.74 \\
\hline 3 & 0.89 & 1.01 & -0.11 & -0.43 & 108 & 0.67 & 1.05 & 0.30 \\
\hline 3 & -0.83 & -0.60 & -0.22 & -0.95 & 108 & 0.34 & 1.24 & 0.26 \\
\hline 3 & 0.88 & 1.27 & -0.38 & -1.37 & 108 & 0.17 & 1.56 & 0.21 \\
\hline 3 & 0.81 & 1.13 & -0.32 & -1.23 & 108 & .2216 & 2.82 & 0.09 \\
\hline 3 & 0.83 & 1.03 & -0.20 & -0.75 & 108 & .4521 & 0.03 & 0.95 \\
\hline
\end{tabular}

Nota: MNSQ: Mínimos Cuadrados Normados; RSME: Raíz Cuadrada del Error Cuadrático Medio; Diferencia de las medidas: Diferencia measure 1; Diferencia del contraste: mayor a 0.5; Índice de RaschWelch; Índice Manten Haenzel; Prob: probabilidad esperada inferior a 0.05 
Tabla 7. Funcionamiento diferencial ítems respecto a la condición clínica, la severidad de la depresión (depresión mayor) y el género

\begin{tabular}{|c|c|c|c|c|c|}
\hline \multirow[b]{2}{*}{ Ítem } & \multirow[b]{2}{*}{ Enunciado } & \multicolumn{4}{|c|}{ Discriminativos Depresión } \\
\hline & & $\begin{array}{l}\text { Condición } \\
\text { clínica }\end{array}$ & $\begin{array}{l}\text { Depresión } \\
\text { Mayor }\end{array}$ & Mujeres & Hombres \\
\hline 11 & Cambia muy a menudo de pareja & & $\mathrm{x}$ & & \\
\hline 12 & Ha deseado abandonar todo & $\mathrm{x}$ & & $\mathrm{x}$ & \\
\hline 17 & $\begin{array}{l}\text { Ha buscado experimentar sensaciones extremas aun cuando ponga en } \\
\text { riesgo su vida }\end{array}$ & $\mathrm{x}$ & $\mathrm{x}$ & $\mathrm{x}$ & \\
\hline 22 & Busca estar ocupado haciendo actividad física & $\mathrm{x}$ & & & $\mathrm{x}$ \\
\hline 28 & Consume alcohol hasta embriagarse & $\mathrm{x}$ & & & $\mathrm{x}$ \\
\hline 30 & Se comporta de tal manera que los demás piensan que parece otra persona & $\mathrm{x}$ & & & $\mathrm{x}$ \\
\hline
\end{tabular}

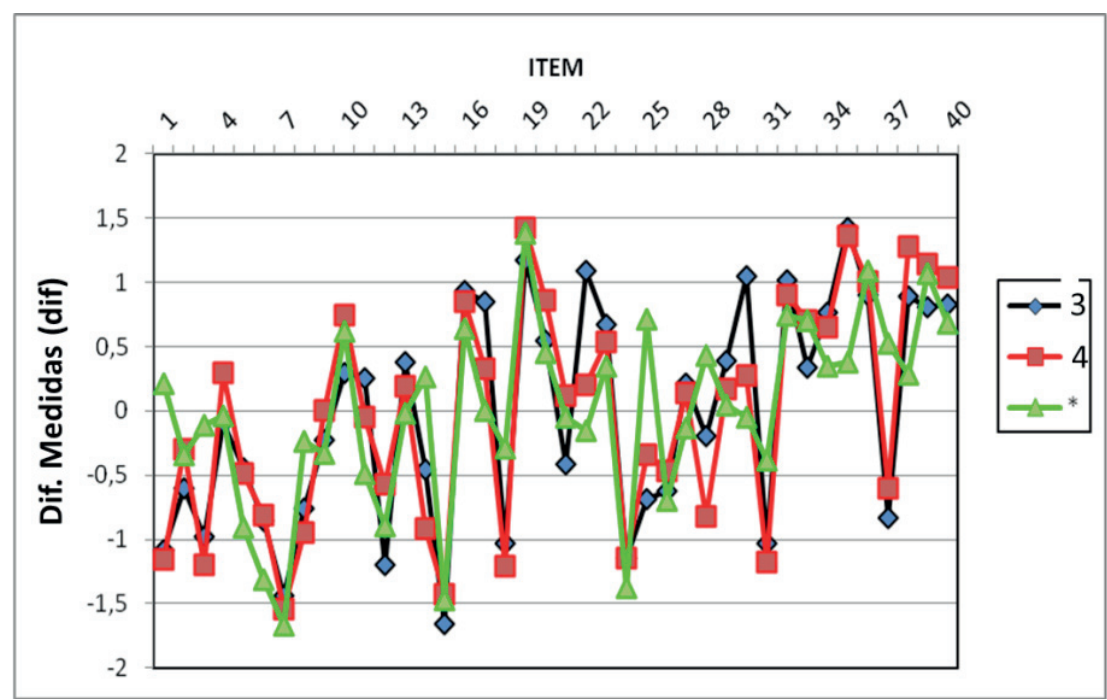

Notas: p Dif: Diferencia significativa de las medidas obtenidas por los grupos en cada ítem

Figura 2. Funcionamiento diferencial de los items entre hombres $(n=59)$ y mujeres $(n=59)$ con depresión.

En la Figura 2 se evidencia que los ítems 2, 4, 9, 12, 16, $19,20,21,24,27,29,32,37$ y 38 se presentan más ajustados a la población masculina deprimida (línea roja) que a la población femenina (línea azul), pero las diferencias solo son significativas en los ítems 12, 17, 28, 30 y 22.

Para determinar la validez concurrente se analiza la covariación entre los puntajes obtenidos en el MINI (variable criterio) y el CDH y se halló una correlación de Pearson de .813 con un $\mathrm{p} \leq .001$ entre estas escalas y de Pearson de .733 con un p menor o igual a .001 entre las escalas de riesgo suicida.

Adicionalmente, se encontraron correlaciones de Pearson significativas entre el Factor 1 del MINI (severidad) y el Factor 2 del CDH (Ideación suicida); y entre los Factores del CDH 4 (Afecto negativo), 3 (Pobre imagen social) y 1 (Autoimagen negativa), y el Factor 1 del MINI (Severidad); y entre el Factor 3 del MINI (Riesgo suicida) y el Factor 2 del CDH (Ideación suicida) (Tabla 8).

En la Curva COR presentada (Figura 3) se observa que el modelo es posiblemente optimo, ya que el CDH tiene un nivel de sensibilidad (verdaderos positivos VPR) y especificidad (falsos positivos FPR) cercano al del MINI de Depresión considerada hasta ahora la medida de oro. Y los casos no detectados adecuadamente eran de mujeres. El área bajo la curva de rendimiento diagnóstico indica el porcentaje de especificidad de $0.98 \%$ que se acerca a 1 considerado el valor diagnóstico perfecto. 
En la Figura 4 se evidencia que los participantes tienen resultados similares en ambas pruebas y que la inclinación de la pendiente no es pronunciada; las ubicaciones de los

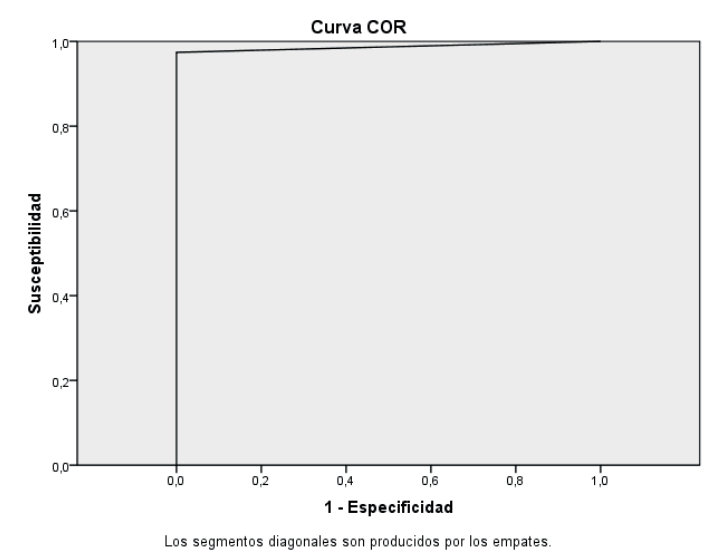

Verdaderos positivos 116 Falsos positivos 0

Falsos negativos $0 \quad$ Verdaderos negativos 110

Área bajo la curva 0.98

Figura 3. Sensibilidad y especificidad COR del CDH respecto del MINI de depresión. participantes pueden ser representadas usando una línea recta, aunque se evidenció la existencia de casos atípicos que habían sido previamente detectados.

\section{Calificación}

Se ajustó la calificación del $\mathrm{CDH}$ a partir de un análisis estadístico confirmado con el criterio clinico de 5 expertos ( 2 psiquiatras y 3 psicólogos con estudios de doctorado) quienes revisaron el resumen de los casos clínicos y no clínicos, de ambos grupos se presentaron las calificaciones obtenidas en el CDH y el MINI-Depresión. Luego se definió en consenso que el punto clínico, y se reconfirmaron los puntos de corte de cada dimensión. Así se estableció que el punto clínico de corte de 70 y se definió que puntajes entre 70 y 99 indicaban la existencia de depresión leve (subclinica/en recuperación), entre 100 y 119 de depresión moderada, de 120 hacia arriba depresión severa. Además se definió que la calificación de cada dimensión se obtiene sumando los puntajes alcanzados en cada uno de los ítems que la componen.

Una vez analizados los resultados se decidió la versión final de los ítems incluidos en el CDH (Tabla 9).

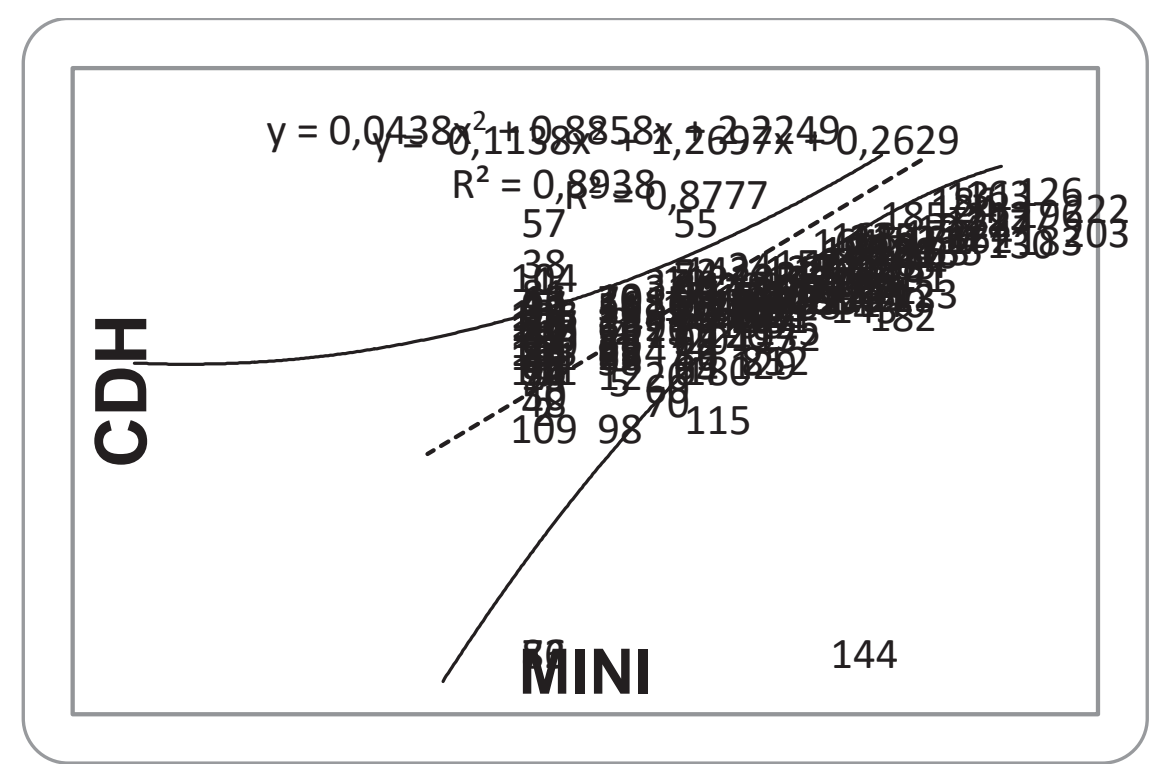

Figura 4. Covariación de los puntajes obtenidos en el MINI y el $\mathrm{CDH}$ 
Tabla 9. Ítems incluidos en la versión final del CDH

\begin{tabular}{|c|}
\hline AFIRMACIÓN \\
\hline 1. Se siente agotado \\
\hline 2. Ha sentido que tiene pocas opciones de realizarse como persona en la vida \\
\hline 3. No imagina cómo será su vida dentro de 10 años \\
\hline 4. Se siente vacío \\
\hline 5. Tiene dificultad para controlar sus emociones \\
\hline 6. Se siente inquieto \\
\hline 7. Tiene problemas para dormir bien \\
\hline 8. Se siente irritable \\
\hline 9. Se ha sentido intranquilo especialmente en la mañana \\
\hline 10. Ha sentido pesar de sí mismo \\
\hline 11. Ha tenido relaciones de pareja paralelas \\
\hline 12. Ha deseado abandonar todo \\
\hline 13. Se siente insatisfecho con lo que ha hecho de su vida \\
\hline 14. Reacciona agresivo ante situaciones normales \\
\hline 15. Se siente estresado \\
\hline 16. Presiente que su futuro va a ser más malo que bueno \\
\hline 17. Ha buscado experimentar sensaciones extremas aun cuando ponga en riesgo su vida \\
\hline 18. Se siente cansado sin razón \\
\hline 19. Ha pensado en formas para terminar con su vida \\
\hline 20. Se le dificulta tomar decisiones cotidianas \\
\hline 21. Se siente impaciente \\
\hline 22. Busca estar ocupado para no pensar \\
\hline 23. Ha pensado que los demás estarían mejor sin usted \\
\hline 24. Se ha sentido ansioso especialmente en la mañana \\
\hline 25. Siente que no sirve para nada \\
\hline 26. Ha tenido cambios bruscos en su estado de ánimo \\
\hline 27. Siente que es mejor evadir dificultades haciendo otras actividades con sus amigos \\
\hline 28. Consume alcohol hasta embriagarse \\
\hline 29. Se siente frustrado \\
\hline 30. Se comporta de tal manera que los demás piensan que parece otra persona \\
\hline 31. Ha sentido que otros lo ven como apagado \\
\hline 32. Usa palabras como incapaz e inútil para definirse \\
\hline 33. Ha sentido que otros lo ven como pesimista \\
\hline 34. Siente insatisfacción con lo que hace \\
\hline 35. Se queja para conmover a otros y obtener apoyo \\
\hline 36. Ha deseado terminar con su vida para no sufrir más \\
\hline 37. Fantasea con dormir y no despertar \\
\hline 38. Le han dicho que es una persona difícil de tratar \\
\hline 39. Ha deseado morir para solucionar sus problemas \\
\hline 40. Suele demeritar lo que hace \\
\hline
\end{tabular}




\section{Discusión y conclusiones}

Una vez analizados los resultados es posible afirmar que el $\mathrm{CDH}$ cumple los tres supuestos principales de la teoría de respuesta al ítem unidimensionalidad, porque se mide un único rasgo latente o dimensión dominante (rasgo principal depresión), que se expresa a través de otras subdimensiones no dominantes pero que son componentes del constructo (autovaloración negativa, ideación suicida, afecto negativo, pobre imagen social, desesperanza y evitación); además cumplió el criterio de independencia local de las respuestas porque cada nueva respuesta era independiente de las repuestas dadas previamente por los evaluados, es decir, que no se dio un patrón de respuesta (los casos que lo presentaron fueron excluidos) y la respuesta solo estaba determinada por el contenido del ítem y el grado en que la persona cumplía el aspecto evaluado o nivel de aptitud general (depresión). Además, todos los sujetos con el mismo nivel de aptitud tenían la misma probabilidad de responder de la misma manera y las respuestas dadas por los sujetos a cada ítem no se influencian mutuamente.

La unidimensionalidad identificada en el Cuestionario de Depresión en Hombres (CDH) es considerada un importante indicador de su validez, por cuanto denota que los 40 ítems que lo conforman, en conjunto miden un constructo global o rasgo denominado depresión; es decir, que este mismo constructo resulta suficiente para explicar las distintas respuestas dadas por los participantes ante dicho conjunto de ítems. Además, está el hecho de que el ajuste de los ítems, evaluado a través de los INFIT y OUTFIT, es apropiado en la mayor parte de los casos (Hambleton y Jones 1993), y que en los casos que no fue así una vez detectado se procedió a modificarlos para luego evaluarlos en la aplicación nacional del instrumento.

El CDH a la vez cumple con el criterio de separación que indica que las respuestas entre los distintos ítems son estadísticamente independientes, es decir, que las respuestas dadas a cada uno de los ítems no se afectan mutuamente, ni dependen una de otras (Linacre, 2002). Como parte de las pruebas realizadas para determinar la validez del cuestionario se analizó el Funcionamiento Diferencial de los Ítems (DIF), que permitió identificar la existencia de sesgos de medida debidos a variables específicas de clasificación de grupos, como el género en este caso; para ello se evaluó el DIF de los ítems respecto de los dos grupos de personas deprimidas, y se comparó el funcionamiento específico de los ítems en mujeres y en hombres.

La teoría de respuesta al ítem facilita el desarrollo de estudios psicométricos con grupos específicos de difícil acceso y consecución, como en el caso de la depresión, ya que gracias a la objetividad específica que garantiza el ajuste de los datos y a que el modelo de medida es independiente de la muestra de sujetos y no se requiere de grandes muestras. Aunque si se requiere un número equilibrado de sujetos con el mismo nivel de expresión de la variable para lograr un ajuste total de la medida y en la muestra objeto de estudio había un número reducido de casos severos de depresión, por tanto, es importante dirigir los esfuerzos hacia el estudio del funcionamiento del instrumento en casos de alta severidad.

De otra parte, los ítems que presentaron dificultades de ajuste fueron revisados teniendo en cuenta los reportes dados por algunos participantes luego de una entrevista realizada a hombres y mujeres que conformaban los distintos grupos de participantes dirigida a identificar las dificultades existentes en los ítems. Se encontró que tenían limitaciones en su redacción que afectaban el ajuste y por ello fueron modificados. Todo porque se pretende conservarlos en la prueba para analizar su funcionamiento en el segundo estudio, que además permitirá reconfirmar la validez del instrumento.

También se encontró que dos ítems -que evaluaban el cambio a menudo de pareja y la búsqueda de sensaciones extremas ignorando el riesgo vital- presentaban funcionamiento diferencial en los casos más severos, y que si quien los responde marca en ambos respuestas positivas de mayor valencia se espera que obtenga una calificación global indicativa de alta severidad. En otras palabras estos ítems tienen mayor probabilidad de ser respondidos afirmativamente y con mayor grado de intensidad si la persona exhibe un cuadro depresivo mayor (Atorresi, Lozzla, Abal, Galiberty Aguerri, 2009). Pero no se puede asumir que estos ítems conforman una escala de severidad, ya que este atributo debe ser medido teniendo en cuenta tanto la intensidad de los síntomas como el grado de afectación producido por ellos en el desenvolvimiento cotidiano de las personas, tema que debe ser motivo de nuevos desarrollos de este cuestionario.

La importancia de incluir en los instrumentos escalas que valoren la severidad había sido previamente reconocida por Ruiz, Silva y Miranda (2001) pero no se cuenta con estudios dirigidos a analizar el funcionamiento diferencial de los ítems en población colombiana y solo se ha realizado este análisis para evaluar el sesgo métrico debido al género y al tipo de trastorno en la escala BDI-II en España (Colegio Oficial de Psicólogos, 2011), pero hace falta corroborar este funcionamiento en otras poblaciones.

En suma, la respuesta dada a ciertos ítems no dependía estrictamente del nivel de depresión expresado, sino que estaba mediada por el género, y es posible pensar que estos ítems no pueden ser usados con equidad para medir 
depresión en hombres y mujeres, y que en el ámbito clínico deberían usarse únicamente para detectar y medir depresión en hombres.

Un indicador de la adecuada discriminación lograda por el CDH fue la confirmación del punto de corte calculado a partir de métodos estadísticos por un grupo de expertos en depresión; ya que no solo era importante alcanzar significancia estadística, también era preciso ajustar las medidas a criterios clínicos que le dieran sentido y sustento a los indicadores numéricos hallados. No se puede perder de vista que la psicología de las personas va más allá del manejo de cifras y requiere de la sensibilidad propia de quienes se desenvuelven día a día como terapeutas (Iraguri, 2009), que van más allá pueden reconocer la importancia de medidas sensibles e importantes como las del sufrimiento humano $\mathrm{y}$ aquellas que pueden ayudar a prevenir suicidios.

Los resultados obtenidos en el CDH tenían alta concordancia con los obtenidos en la entrevista MINI, usada como prueba de oro debido a su valor predictivo del $75 \%$ y grado de sensibilidad y especificidad del 85\% (American Psyquiatryc Association, 2001; Pinnintim et al., 2003); pero dicha relación no se mantiene para los casos más severos, probablemente porque los procesos evaluativos resultan ser más complejos debido a la riqueza de los síntomas y solo a través del uso de técnicas de heteroevaluación como la entrevistas diagnosticas MINI es posible recabar información más completas acerca del caso.

De modo tal que facilita a los profesionales establecer un dictamen de tipo diagnóstico. En general en las pruebas de autorreporte ya se han reconocido previamente limitaciones en los alcances evaluativos ya que dependen de una parte de la capacidad de auto observación que tiene quien responde, de su deseo de ser sinceros y de la comprensión misma de las cuestiones abordadas en las preguntas.

De otra parte, la concordancia del CDH con los diagnósticos emitidos por algunos profesionales fue menos estricta (por ejemplo, 5 casos en los que se hizo una clasificación más específica del tipo de trastorno a partir de los resultados obtenidos en los instrumentos), esto puede ser porque los profesionales remisores incluyeron una etiqueta diagnóstica general de depresión especialmente en los casos de depresión en hombres. Probablemente el reducido conocimiento sobre la depresión masculina o la falta de sistematización del mismo, hace que el profesional emita un juicio global derivado de su observación, experiencia y experticia clínica, que, del uso estricto del sistema para diagnosticar estos casos, y ello dificulta la identificación de ciertas características específicas del trastorno.
La alta coincidencia de los resultados del CDH y los obtenidos en la entrevista MINI, previamente hallados en otros estudios realizados en población colombiana (Álvarez y Londoño, 2012; Vargas et al., 2013); así como la concordancia entre los resultados obtenidos en las escalas relacionadas con riesgo e ideación suicida apuntan hacia la posibilidad de usar el $\mathrm{CDH}$ en procesos de cribado, como fuente de reconfirmación diagnóstica y en investigación adelantada en el campo de la psicología clínica en hombres.

No obstante, la concordancia no estricta en la detección de casos de depresión mayor, tanto en hombres como en mujeres, limita el uso del CDH e indica que es preciso realizar nuevos estudios dirigidos a mejorar el funcionamiento de los ítems y su poder discriminativo en casos de depresión mayor, pues aunque en la teoría de respuesta al ítem no se requieren tamaños muéstrales mayores para adelantar este tipo de análisis, la baja participación de personas diagnosticadas con Depresión Mayor no permitió llevar a cabo este procedimiento.

\section{Limitaciones y futuras direcciones}

A pesar de que se realizó entrenamiento conjunto de los auxiliares y asistentes de investigación, la aplicación de los cuestionarios fue llevada a cabo en condiciones cualitativamente distintas y por profesionales con diversos niveles de entrenamiento con mayor o menor habilidad para estimular el reporte abierto de sus síntomas; entonces en nuevos trabajos y para controlar de alguna forma ésta variable puede delimitarse el tiempo mínimo de experiencia y un nivel mínimo de formación exigido a fin de intentar unificar el nivel de competencia profesional y conocimiento de quienes remiten y entrevistan. Finalmente, aún es preciso desarrollar una versión breve del Cuestionario que le dé mayor utilidad clínica e investigativa al CDH y determinar escalas específicas por género, así como escalas de síntomas centrales de depresión.

\section{Referencias}

Ackerman, T. A. (1992). A didactic explanation of item bias, item impact, and item validity from a multidimensional perspective. Journal of Educational Measurement, 29, 67-91.doi: 10.1111/j.1745-3984.1992. tb00368.x.

Álvarez, N., y Londoño, C. (2012). Diseño y validación del Cuestionario de Depresión en Hombres. Tesis de Maestría. Universidad Católica de Colombia.

American Psychiatric Association. (2001). Diagnostic and statistical manual of mental disorders, DSM-IV TR. Washington, D. C.: Masson. Atorresi, I., Lozzla, G., Abal, F., Galibert, S., y Aguerri, M. (2009). Teoría de Respuesta al Ítem: conceptos básicos. Revista Argentina de Psicologia 
Clinica, 18, 179-188. Recuperado desde www.clinicapsicologica.org. ar/download_file.php?idt $=123$

Besharat, M. A., Addolmananfi, A., Farahani, H., y Khodaii, M. (2011). The moderating role of locus of control on the relationship between anger and depression in patients with major depression disorder. Procedia-Social and Behavioral Science, 30, 302-306. doi: 10.1016/j. ajp.2012.07.013

Beusenberg, M. y Orley, J. (1994). A user's guide of the Self Reporting Questionnaire. World Health Organization. Recuperado de: http://apps. who.int/iris/bitstream/10665/61113/1/WHO_MNH_PSF_94.8.pdf

Brownhill, S., Wilhelm, K., Barclay, L., y Schmied, V. (2005). Big build: hidden depression in men. Australian and New Zeland Journal of Psychiatry, 39, 921-931. doi: 10.1111/j.1440-1614.2005.01665.x

Chang, S. M., Hahm, B-J., Lee, Y-J., Shin, M.S., Jeon, H. J., Hong, J. P., y Cho, H. (2008). Cross-national difference in the prevalence of depression caused by the diagnostic threshold. Journal of Affective Disorders, 106, 159-167. doi: 10.1016/j.jad.2007.07.023.

Cochran, S. V., y Rabinowitz, F. E. (2003). Gender-sensitive recommendation for assessment and treatment of men. Professional Psychology: Research and Practice, 34, 132-140. Recuperado desde http://www. apa.org/research/-action/men.aspx

Colegio Oficial de Psicólogos España-COP (2011). Evaluación del inventario BDI- II. Recuperado desde http://www.cop.es/uploads/ PDF/2013/BDI-II.pdf

Galicia, I., Sánchez, A., y Robles, P. J. (2009). Factores asociados a la depresión en adolescentes. Anales de Psicología, 25, 227-240. doi: $10.6018 / 87501$

Gonçalves, D., Stein, A. y Kapczinski, F. (2008). Avaliação de desempenho do Self-Reporting Questionnaire como instrumento de rastreamento psiquiátrico: um estudo comparativo com o Structured Clinical Interview for DSM-IV-TR. Cadernos. Saúde Pública, 24 (2). 380-390. Recuperado de: http://www.scielo.br/pdf/csp/v24n2/16.pdf

Gómez, J., y Navas, M. J. (1998). Impacto en el funcionamiento diferencial de los ítems respecto al género. Psicothema, 10, 685-696. Recuperado desde http://www.psicothema.com/pdf/197.pdf

Hambleton, R., y Jones, R. (1993). Comparison of classical test theory and item response theory and their applications to test development. Education of Measure, 120, 38-47. doi: 10.1111/j.1745-3992.1993.tb00543.x

Hewitt, P. L., y Flett, G. L. (1991). Perfectionism in the self and social contexts: conceptualization, assessment, and association with psychopathology. Journal of Personality and Social Psychology, 60, 456-470. doi: 10.1037/0003-066X.38.11.1145

Iraguri, I. (2009). Evaluación de los resultados clínicos: Entre la significación estadística y la relevancia clínica. Norte de Salud Mental 33, 94-108. Recuperado desde http://antigua.ome-aen.org/norte/33/ NORTE $33 \quad 140$ 94-108.pdf

Joormann, J., y Gotlib, I. H. (2010). Emotion regulation: relation to $\operatorname{cog}$ nitive inhibition. Cognition and Emotion, 24, 281-298. doi: 10.1016/j. ajp.2012.07.013

Kilmartin, Ch. (2005). Depression in men: communication, diagnosis and therapy. Journal of Men's Healthand Gender, 2, 95-99.doi: $10.1080 / 17482620701547008$

Linacre, J. M. (2002). What do Infit and Outfit, Mean-square and Standardized mean? Rasch Measurement Transaction, 168, 878.Recuperado de: http://www.rasch. org/rmt/rmt162f.htm)

Millsap, R. E., y Everson, H. T. (1993). Methodology review: Statistical approaches for assessing measurement bias. Applied Psychological Measurement, 17, 297-334.doi: 10.1177/014662169301700401

Mittendorfer-Rutz W. E. (2006a). Social psychiatry and public mental health: present situation and future objectives: Time for rethinking and renaissance? Acta Psychiatric Scandinavian, 3, 95-100.

Mittendorfer-Rutz, W. E. (2006b). Trends of youth suicide in Europe during the 1980's and 1990's.Gender Differences and Implications for Prevention, 3, 250-257. doi: 10.1016/j.jmhg.2006.02.006.

Möller-Leimkühler, A. M., y Yücel, M. (2010). Male depression in females? Journal of Affective Disorders, 121, 22-29. doi: 10.1016/j. jad.2009.05.007
Möller, A.M., Heller, J., y Paulus, N. C. (2006). Subjective well-being and 'male depression' in male adolescents a Department of Psychiatry, Journal Affects Disorders, 7, 65-72. doi: 10.1016/j.jad.2006.07.007

Organización Mundial de la Salud. (2009). Gender and Mental Health. Ginebra: Organización Mundial de la Salud. Recuperado desde http:// www.Who.int/gender/henderandhealth.html.

Organización Panamericana de la Salud. (2012). Día Mundial de la Salud Mental. Recuperado desde http://www.paho.org/arg/index. php?option $=$ com content\&view $=$ article $\&$ id $=1047 \&$ Itemid $=325$

Paykel, E. S. (1991). Depression in women. British Journal of Psychiatry, $158,22-29$

Pinnintim, N., Madison, H., Musser, E., y Rissmiller, D. (2003). MINI International Neuropsychiatric Schedule: clinical utility and patient acceptance. European Psychiatry, 18, 361-364. doi: S0924933803001159

Pollack, W., Garbarino, J., Kindlon, D., y Thompson, M. (1999). Boys to men: Emotional miseducation. American Psychological Association Monitor, 30, 38-39. Recuperado desde http://www.fact.on.ca/newpaper/ am9907.htm

Rodié, J., y Usall, I. (2001). Diferencias de género en los trastornos del estado de ánimo: una revisión de la literatura. Centro de Salud Mental. El Prat de Llobregat. Barcelona.

Rude, S. S., Chrisman, J. G., Denmark, A. B., y Maestas, K. L. (2012). Expression of direct anger and hostility predict depression symptoms in formerly depressed women. Canadian Journal of Behavioral Science, 44, 200-209..doi: 10.1037/a0027496

Ruiz, A., Silva, H., y Miranda, E. (2001). Diagnóstico clínico y psicométrico de la depresión en pacientes de medicina general. Revista Médica de Chile, 129. doi: 10.4067/S0034-98872001000600005

Sheehan, D. V., Lecrubier, Y., Sheehan, K. H., Amorim, P., Janavs, J., Weiller, E., Herqueta, T., Baker, R., y Dunbar, G.C. (1988). The MiniInternactional Interview (M.I.N.I.): The development and validation of a structure diagnostic psychiatric interview for DSM-IV and ICD-10. Journal of Clinical Psychiatry, 59, 22-33.

Vargas, J., Ruiz, J., Hernández, L. M., y Londoño, C. (2013). Depresión y masculinidad en hombres de la ciudad de Ibagué. Tesis de Pregrado. Coruniversitaria de Ibagué. Ibagué, Colombia.

Wilhelm, K., Boyce, P., y Brownhill, S. (2004). The relationship between interpersonal sensitivity, anxiety disorders and major depression. Journal of Affective Disorders, 79, 33-41. doi10.1016/S01650327(0200069-1

Worley, M., Trim, R., Roesch, S., Mrnak-Meyer, J., Tate, S., y Brown, S. (2012). Comorbid depression and substance use disorder: Longitudinal associations between symptoms in a controlled trial. Journal of Substance Abuse Treatment, 43, 291-302.doi: 10.1016/j.jsat.2011.12.010

Zierau, F., Bille, A., Rutz, W. y Bech, P., (2002). The Gotland Male Depression Scale: A validity study in patients with alcohol use disorders. Nordic Journal of Psychiatry, 56, 265-271. Recuperado de: http:/ www.journals.elsevierhealth.com/periodicals/jmhg/article/S15718913(05)00092-0/references 